\title{
Intrinsic Variability of Ocular Dominance Column Periodicity in Normal Macaque Monkeys
}

\author{
Jonathan C. Horton and Davina R. Hocking \\ Beckman Vision Center, University of California, San Francisco, San Francisco, California 94143-0730
}

\begin{abstract}
Little is known about intrinsic variation from animal to animal in the periodicity of columnar systems within various regions of the mammalian cerebral cortex. To address this issue, complete mosaics of the ocular dominance columns were reconstructed from flat-mounts of the left and right striate cortex (V1) in six normal adult macaques (Macaca fascicularis). To identify the columns, we enucleated the right eye and subsequently processed striate cortex for cytochrome oxidase (CO) activity. Average column areas for the intact eye and the missing eye were nearly equal, confirming that monocular enucleation in adult macaques produces negligible column shrinkage. The contralateral eye's columns occupied more territory than the ipsilateral eye's columns, even in the central visual field representation $\left(0^{\circ}\right.$ to $\left.8^{\circ}\right)$, where they predominated by 52 to $48 \%$. The column mosaics showed remarkable variation in periodic-
\end{abstract}

In many species, the geniculocortical afferents serving the right and left eyes terminate in a pattern of dovetailed stripes in layer IVc of striate cortex (V1) called ocular dominance columns (Hubel and Wiesel, 1969; Wiesel et al., 1974). In 1985, LeVay and colleagues reconstructed the entire mosaic formed by these columns in macaque striate cortex. They found that the columns give rise to an elaborate system of roughly parallel bands, laid out in a stereotypic fashion with respect to the retinotopic map in V1. Because reconstruction of the complete pattern of columns was such an arduous task, they examined only a single animal. Limiting their study to just one monkey left unanswered the question of how much the column pattern varies from animal to animal. Is the layout of the columns governed by the same rules in all macaques? How much natural variation occurs among animals in the geometry and periodicity of the columns? What might this variation tell us about the process responsible for the formation of the columns, or about the purposes they serve?

Since the first report by LeVay and colleagues (LeVay et al., 1985), subsequent studies have described the complete pattern of ocular dominance columns in a variety of primate species (Rosa et al., 1988, 1992; Horton et al., 1990; Florence and Kaas, 1992). In each of these reports, as in LeVay's original study, complete reconstruction of the columns was accomplished in only a single specimen. Moreover, investigators differed to some extent in how they fixed the tissue, labeled the ocular dominance columns, and

Received June 14, 1996; revised Aug. 29, 1996; accepted Sept. 4, 1996.

Correspondence should be addressed to Dr. Jonathan C. Horton, Beckman Vision Center, 10 Kirkham Street, University of California, San Francisco, San Francisco, CA 94143-0730.

This work was supported by grants from the National Eye Institute, That Man May See, Inc., and Research to Prevent Blindness. We thank Dr. Dale Purves and Dr. Geoffrey J. Goodhill for their critical review of this manuscript.

Copyright (C) 1996 Society for Neuroscience $\quad 0270-6474 / 96 / 167228-\bullet \$ 05.00 / 0$ ity. The number of column pairs along the V1/V2 border ranged from 101 sets in one monkey to 154 sets in another. Average column width along the V1/N2 border ranged between 670 and $395 \mu \mathrm{m}$, a nearly twofold difference. The widest columns were found in the foveal representation. This high degree of innate variability should be taken into account when considering the effects of various sensory manipulations (e.g., strabismus, anisometropia), which have been reported to alter the periodicity of ocular dominance columns. We found pronounced intrinsic variation in the width and number of ocular dominance columns in a sample of six $M$. fascicularis, indicating that the number of hypercolumns within a given cortical area can range widely among normal members of the same species.

Key words: ocular dominance column; visual cortex; cytochrome oxidase; macaque; flat-mount; periodicity; hypercolumn

reconstructed their pattern. For these reasons, the findings in these studies are difficult to compare. They leave unsettled the basic question of how much intrinsic variation occurs in the pattern of columns from animal to animal in a single species.

Our interest in this problem was stimulated by several reports that the periodicity of ocular dominance columns is affected by raising animals with strabismus, anisometropia, or alternating monocular occlusion (Löwel, 1994; Roe and Ts'o, 1995; Tieman and Tumosa, 1996). To aid in the interpretation of these studies, we thought it might be pertinent to report our experience gained from the study of ocular dominance columns in normal animals. Over the past 3 years, we have prepared complete flat-mounts of the ocular dominance columns in six normal adult macaques (Macaca fascicularis). We have been struck by a remarkable variability in the periodicity of the columns, although their general layout is quite consistent from animal to animal. The purpose of this paper is to illustrate the degree of intrinsic variation seen in the periodicity of ocular dominance columns within a small group of normal adult macaques from the same species and to point out certain features of the columns that differ from animal to animal.

\section{MATERIALS AND METHODS}

Experimental animals. Six normal adult cynomolgus macaques ( $M$. fascicularis) were used for these experiments (Table 1). Five shared a common genetic heritage, coming from a feral colony on the island nation of Mauritius. Macaques were introduced to Mauritius in the early 17th century, when they escaped from Dutch sailors keeping them as pets aboard ships bound from Java (Houghton, 1988). With no natural predators, they have proliferated to a population of 50,000-60,000, destroying rare indigenous species and raiding croplands. The minimum ages listed in Table 1 were based on dates of capture, because birthdates were unknown. A single monkey came from a feral colony in the Philippines.

Surgical procedures. Before use, each animal was screened for any preexisting eye disease using standard ophthalmological technique. The 


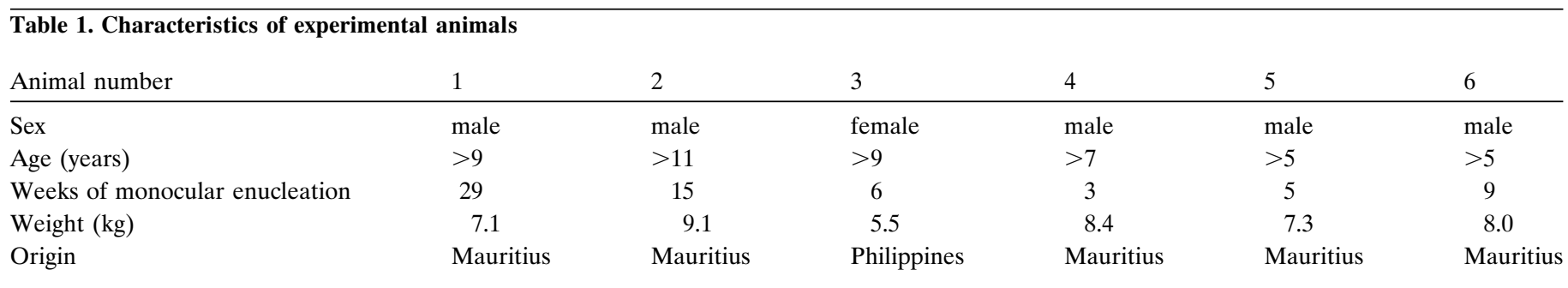

pupils, eye movements, and eye alignment were normal. To test eye alignment, we relied on centration of the corneal light reflex in both pupils of the alert, fixating monkey (Hirshberg test). This method can detect strabismus greater than $3^{\circ}$ to $4^{\circ}$, but not microstrabismus. After ocular motility and alignment were assessed, each animal was sedated (ketamine $\mathrm{HCl}, 20 \mathrm{mg} / \mathrm{kg}$, i.m.) for cycloplegic refraction and fundus examination. No animal had any media opacity, abnormal or unequal refractive error, or strabismus (again, with the proviso that a microstrabismus could not be excluded).

To remove one eye, each monkey was placed under general endotracheal anesthesia with $2.5 \%$ isoflurane and $\mathrm{N}_{2} \mathrm{O} / \mathrm{O}_{2}$ (50:50). Following a protocol approved by the University of California, San Francisco Committee on Animal Research, the right eye was enucleated using sterile technique. After surgery, the animal was treated with buprenorphine $\mathrm{HCl}$ $(0.02 \mathrm{mg} / \mathrm{kg}$, i.m.) every $8 \mathrm{hr}$ for $2 \mathrm{~d}$ to ensure analgesia.

Ocular dominance columns appear within $24 \mathrm{hr}$ of monocular enucleation in cytochrome oxidase (CO)-stained sections of striate cortex. However, maximum contrast in $\mathrm{CO}$ activity is obtained by waiting 3 weeks. Therefore, all animals in this study survived at least 3 weeks (Table 1). Some survived even longer, because they were used for an unrelated study of cortical myelin patterns induced by monocular enucleation (Horton and Hocking, 1997). These differences in survival times do not affect the pattern of ocular dominance columns in adult animals (LeVay et al., 1980) and, therefore, are unrelated to the variation in column periodicity that we found.

Flat-mounting of striate cortex. After receiving a lethal dose of sodium pentobarbital intraperitoneally, each animal was perfused through the left ventricle with 11 of normal saline followed by 1.51 of $2 \%$ paraformaldehyde in $0.1 \mathrm{~m}$ phosphate buffer. The occipital lobes were removed immediately and placed into $0.1 \mathrm{M}$ phosphate buffer. Striate cortex was dissected from each occipital lobe, unfolded, and flattened (Fig. 1). To achieve further flattening, the pial surface of the tissue was placed against a glass slide. A sheet of soft foam was applied to the white matter side, followed by a weight $\left(\sim 8 \mathrm{gm} / \mathrm{cm}^{2}\right)$. The tissue sandwich was left to bathe overnight in $1 \%$ paraformaldehyde plus $30 \%$ sucrose for cryoprotection and additional fixation. To flash-freeze the block for sectioning, the pial surface was placed on a glass slide covered with Saran Wrap while a bag of powdered dry ice was pressed against the white matter. Serial sections were cut at $30 \mu \mathrm{m}$, and alternate sections were reacted for $\mathrm{CO}$ activity (Wong-Riley, 1979). We have shown previously by combining $\left[{ }^{3} \mathrm{H}\right]$ proline transneuronal autoradiography with $\mathrm{CO}$ histochemistry in the same animal that the pale columns labeled in this manner represent the ocular dominance columns serving the enucleated eye (Horton, 1984).

Data analysis. To reconstruct the ocular dominance columns in each animal, every other $\mathrm{CO}$ section was photographed with Plus X-Pan 35 $\mathrm{mm}$ film (Eastman Kodak, Rochester, NY) and developed with Microdol-X (Eastman Kodak). The negatives were scanned into the computer, contrast-reversed, and analyzed using Photoshop 3.0 (Adobe Systems, Mountain View, CA). To start a montage, the single image containing the largest portion of layer IVc was chosen as the "base section." We then outlined layer IVc in the next section with the "lasso" tool. The layer IVc fragments of the column pattern from this section were copied and pasted into a layer overlying the base section. They were rendered semitransparent to align them with the base section by matching prominent blood vessels. They were then restored to normal density and "flattened" onto the base section, extending the mosaic of columns in layer IVc. This process was repeated with each alternate section through the entire series until the montage was complete. The contrast and brightness of the final figures were adjusted to match the montages from each animal, but no other Photoshop features were used to retouch or enhance the images. The seams between each fragment in the montages are hardly visible because they can be seen only at high power.

To make black and white drawings, we created a transparent layer overlying the final montage and traced the borders of the dark columns at high magnification on the monitor using the "pencil" function at a 1 pixel width setting. The dark columns were then flood-filled with black, copied, and pasted onto a white background. To quantify column areas, the black and white drawings were transferred to Mocha (Jandel Scientific, San Rafael, CA). A thresholding function assigned pixels to left eye columns (black, level of gray $=1$ ) or right eye columns (white, level of gray $=256$ ). Intermediate levels of gray were used to quantitate the unreconstructable areas, blind spots, and monocular crescents.

To calculate the sample standard deviations (Table 2), values were averaged for the right and left hemisphere of each animal and then treated as a single datum (i.e., $n=6$ ). This was done because parameters were highly correlated between hemispheres for any given animal. However, the alternative approach of analyzing each flat-mount as an independent datum (i.e., $n=12$ ) resulted in a very similar sample standard deviation for each parameter.

\section{RESULTS}

\section{Variability in column periodicity}

The main purpose of this paper is to show the range in periodicity of ocular dominance columns seen in a consecutive sample of six M. fascicularis examined in our laboratory. To make this point, we have selected for illustration the animal with the widest columns (Figs. $2 A, D$ ), an animal with intermediate columns (Figs. $2 B, E$ ), and the animal with the thinnest columns (Figs. $2 C, F$ ). Black and white drawings of these photomontages are shown in Figure 3. Measurements derived from the drawings are provided in Table 2.

The periodicity of ocular dominance columns can be assessed by various methods, including Fourier analysis (Obermayer and Blasdel, 1993). In the belief that the pictures of the column mosaics speak for themselves, we have kept our approach simple. In each animal, we counted the number of pairs of ocular dominance columns that intersected the perimeter of striate cortex (V1/V2 border; vertical meridian). For example, in Monkey 1 there were 102 column pairs along the V1/V2 border, whereas in Monkey 3 there were 150 column pairs. Next, we measured the perimeter of $\mathrm{V} 1$, excluding the monocular crescent, to calculate the average column width along the V1/V2 border. It ranged from $670 \mu \mathrm{m}$ (Monkey 1, right V1) to $395 \mu \mathrm{m}$ (Monkey 3, right V1). Van Essen et al. (1984) have shown that macaque V1 surface area varies enormously, from 690 to $1560 \mathrm{~mm}^{2}$, with no obvious correlation with body weight. One might expect animals with a larger V1 to have fatter columns or more sets of columns. The monkeys in our series happened to be similar in V1 area, close to the means of 1195 and $1069 \mathrm{~mm}^{2}$ reported in macaques (Van Essen et al., 1984; Purves and LaMantia, 1993). Thus, fluctuation in striate area does not account for the range of column periodicity in Figures 2 and 3.

Looking at Figures 2 and 3, it is apparent that the periodicity of the columns correlates with the intricacy of their mosaic. The widest columns were found in Monkey 1 . Its columns formed a relatively simple pattern of nearly continuous, smooth stripes that swept across the operculum from the vertical meridian to the horizontal meridian. By contrast, Monkey 3 had the thinnest 

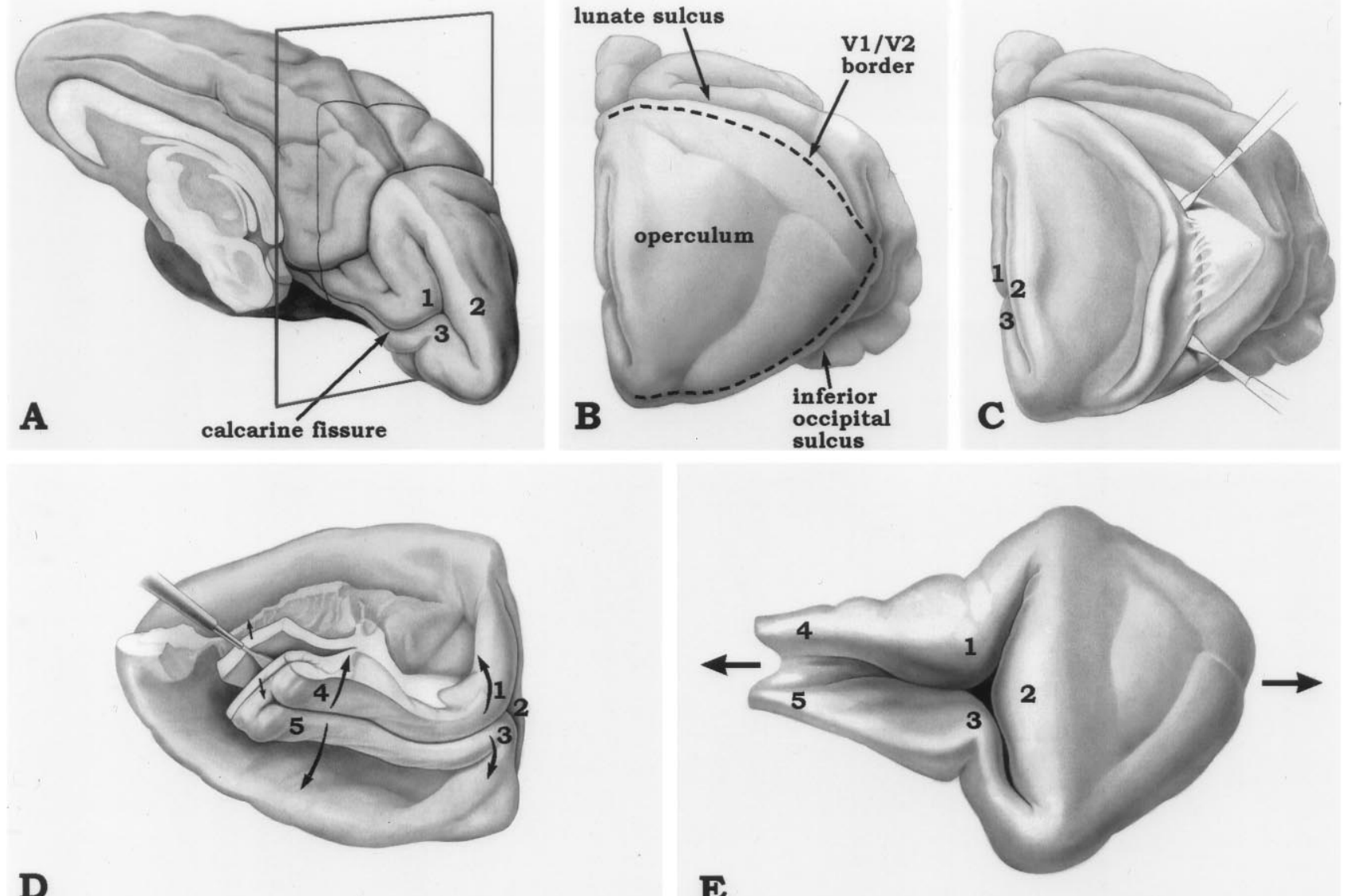

D

$\mathbf{E}$
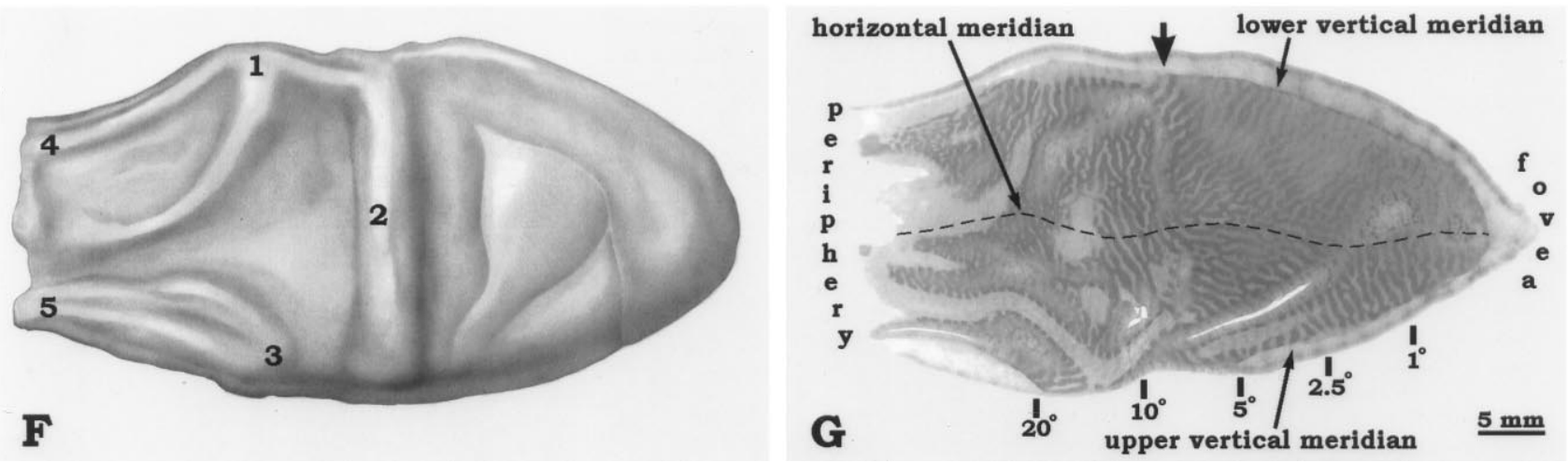

Figure 1. The flat-mounting procedure, shown for the right striate cortex. $A$, The occipital lobe is severed from the brain just behind the splenium of the corpus callosum. $B$, The lunate sulcus and the inferior occipital sulcus, which form the borders of the exposed, flat, posterior surface of the occipital lobe (the "operculum"), are identified and opened by gentle probing with a miniature spatula. $C$, Beginning laterally, the operculum is flayed from the white matter. This process is carried all the way to the midline, creating a free flap of opercular cortex. $D$, Excess tissue is trimmed away, leaving only the opercular flap and the calcarine fissure. The block is then flipped over so that it is sitting on the operculum. A spatula is inserted in the occipital horn of the lateral ventricle and used to open a dissection plane between the cortex and white matter, which is extended along the full length of the calcarine fissure. $E$, The cortex is flipped back to its original orientation, and the calcarine fissure is opened, unfolding striate cortex as a single sheet of tissue. To facilitate this process, it is helpful to insert a moist cotton swab into the calcarine fissure to break pial adhesions. $F$, Most of striate cortex is rendered flat with little distortion by unfolding (if too much occurs, the tissue simply tears). However, the gyri retain some memory of their folded configuration. This tendency is reduced by sandwiching the tissue sheet between a glass slide and a piece of foam overnight. $G$, A single $30 \mu \mathrm{m}$ section stained for CO, showing the ocular dominance columns in Monkey 6 . The section is confined almost entirely to layers III-IV, except along the crests of the gyri, which are still not perfectly flat. The arrow denotes the "hinge," where the operculum folds into the calcarine fissure at the midline. We used a line along this point to divide the operculum $\left(0^{\circ}\right.$ to $\left.8^{\circ}\right)$ from the periphery $\left(8^{\circ}\right.$ to $\left.60^{\circ}\right)$ in making the column measurements in Table 2 . The estimates of eccentricity combine data from Daniel and Whitteridge (1961), Van Essen et al. (1984), and Tootell et al. (1988).

columns. They gave rise to a more complex pattern, with shorter stripes and more frequent fractures, bifurcations, and islands. Despite these differences in column periodicity and mosaic complexity, the ocular dominance columns were laid out according to the same general plan in all animals. The details of this pattern have been described eloquently by LeVay et al. (1985) and will not be reiterated here. It is worth mentioning that although column periodicity varied greatly from monkey to monkey, in any given 


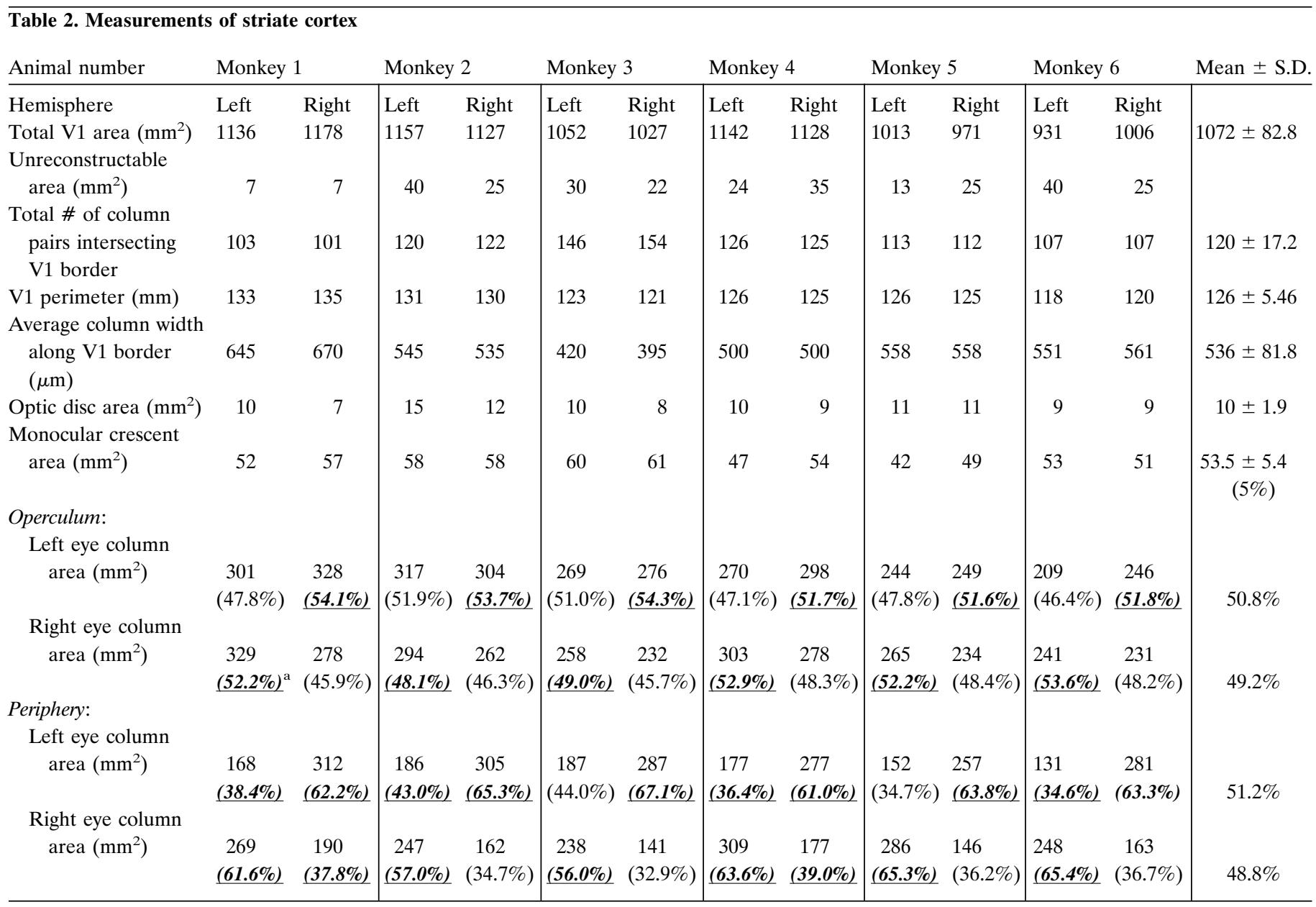

${ }^{a}$ Boldface, underlined italic values denote contralateral eye.

animal the left and right cortices were virtually reciprocal images, with remarkable similarity in size, shape, column periodicity, and mosaic pattern. This resemblance included even minor idiosyncratic features, such as the appearance of the blind spot representation on each side. However, the precise details of the mosaic formed by the columns on each side were not identical.

\section{Larger foveal columns}

Columns were wider along the vertical meridian than along the horizontal meridian for any given eccentricity. This effect was visible in the original column reconstruction illustrated by LeVay et al. (1985; see their Fig. $6 A$ ), although they did not mention it. The period of a column pair also varied with eccentricity: columns were wider within the central field than the peripheral field, as noted previously (LeVay et al., 1985). As a result of these two combined gradients, the foveal representation contained the widest column pairs in each mosaic. This feature was more striking in some animals than in others. Monkey 4, chosen for illustration because it had the most obvious enlargement of foveal columns, is shown in Figures 4 and 5. In a previous study, we pointed out that some animals have unusually large foveal columns (see Fig. 4, Horton and Hocking, 1996). We doubt that expansion of foveal columns is an artifact of cortical stretching during flat-mounting, because the foveal representation is located in a smooth area of opercular cortex that flattens easily with little distortion (Fig. 1). We do not understand the significance of these large foveal columns, or why they are more pronounced in some animals.

\section{Do columns shrink after monocular enucleation in adults?}

In young macaques, monocular enucleation during the critical period of cortical plasticity causes shrinkage of the eye's ocular dominance columns (Hubel et al., 1977). By contrast, no shrinkage of ocular dominance columns is thought to occur after monocular enucleation in adults. This conclusion is based on a single adult macaque studied by LeVay et al. (1980). Four months after monocular enucleation, this animal received an injection of $\left[{ }^{3} \mathrm{H}\right]$ proline into the remaining eye. The ratio of column areas serving the normal and missing eye was 1.0:1 in each hemisphere. However, using CO histochemistry, subsequent investigators have obtained conflicting results. Hess and Edwards (1987) reported a dark/light ratio in column widths of 60:40 in opercular cortex 8.5 months after monocular enucleation in adult Cebus apella. In the same species, 4-7 months after monocular enucleation, Rosa et al. (1992) found a "close to 50\%" ratio of dark and light stripes in opercular cortex. In the macaque, Trusk et al. (1990) noted that "darkly-stained bands in layer $4 \mathrm{C} \beta$ were significantly wider than lightly-stained bands" after monocular enucleation. Finally, in a single M. fascicularis, Florence and Kaas (1992) reported that "CO-dark bands represent $57 \%$ of the paired column width contralateral to the intact eye, and $55 \%$ ipsilateral to the intact eye." However, the age at enucleation was unknown, raising the possibility that their monkey could have been immature and, therefore, vulnerable to column shrinkage. 

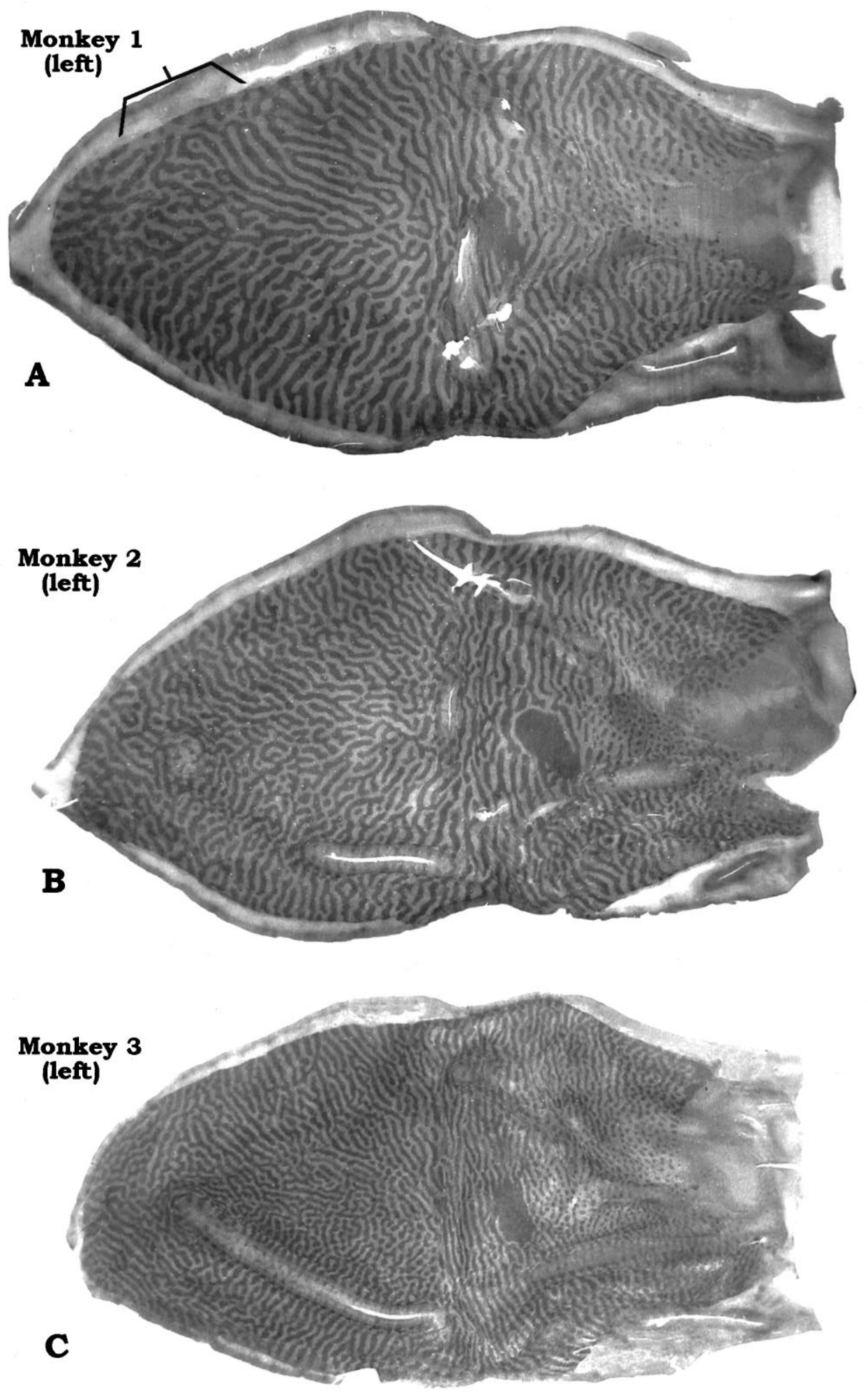

Figure 2. Complete montages of ocular dominance columns from flat-mounts of left and right striate cortex in 3 Macaca fascicularis. Note variation in the periodicity and complexity of the column systems from animal to animal. The bracket in $A$ shows an unusual region where the dark columns (intact left eye) are fragmented and occupy relatively less space than the light columns. 

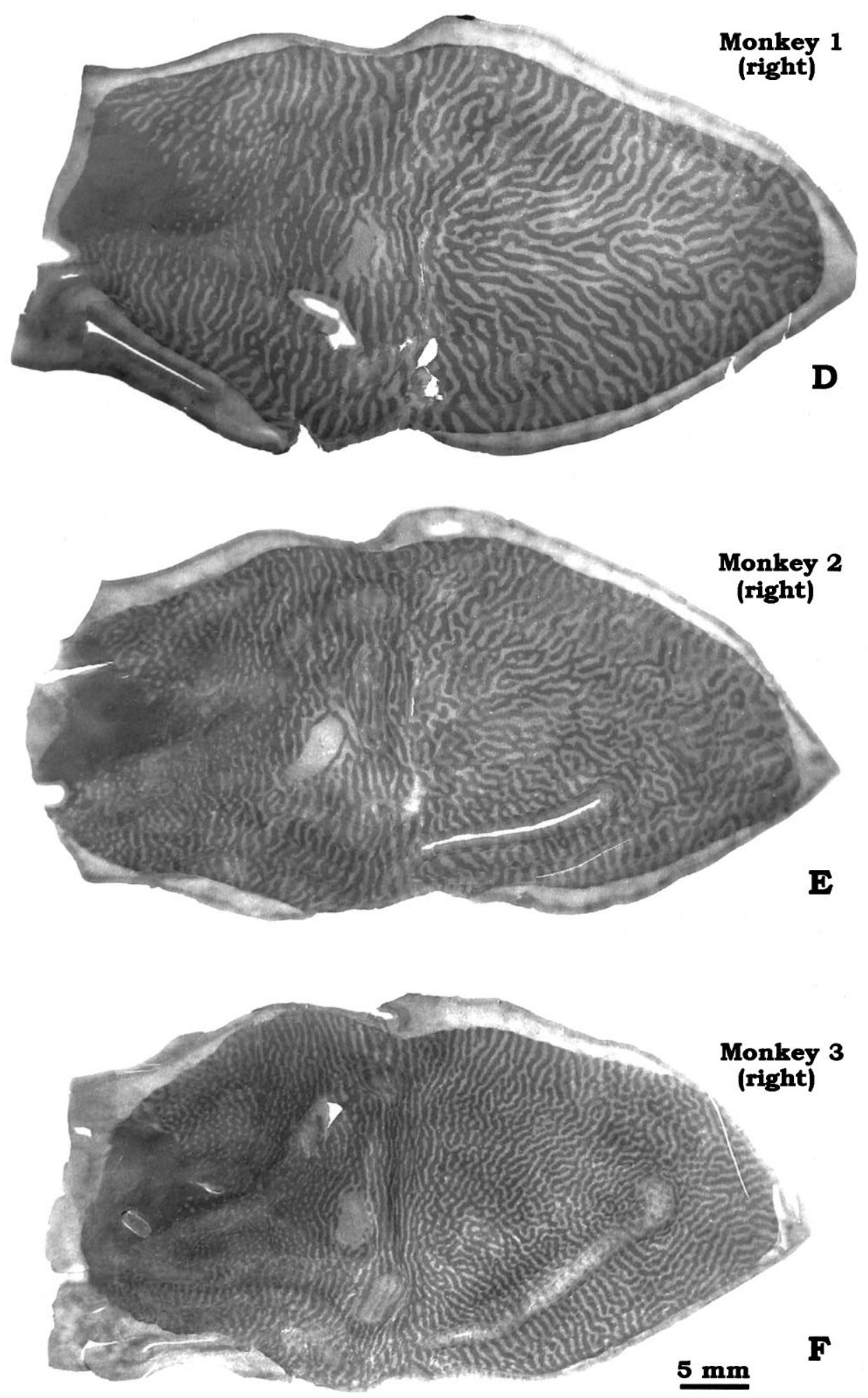

In view of these varied reports, we measured the areas of the dark columns (normal left eye) and light columns (missing right eye) in our mosaics. For opercular cortex, the dark columns averaged $50.8 \%$ and the light columns averaged $49.2 \%$ (Table 2). For peripheral cortex, the areas were $51.2 \%$ dark columns and $48.8 \%$ light columns. Taken at face value, these figures suggest slight shrinkage of the enucleated eye's columns, especially in peripheral cortex. However, there are several reasons we mistrust them. First, the columns are not perfectly sharp, and we might have tended to favor the dark columns when drawing their boundaries. Second, the light columns become difficult to see in peripheral cortex ipsilateral to the enucleated eye, where they appear as small pale islands in a dark sea. By contrast, dark islands in a pale sea on the other side stand out much better. We suspect that this 

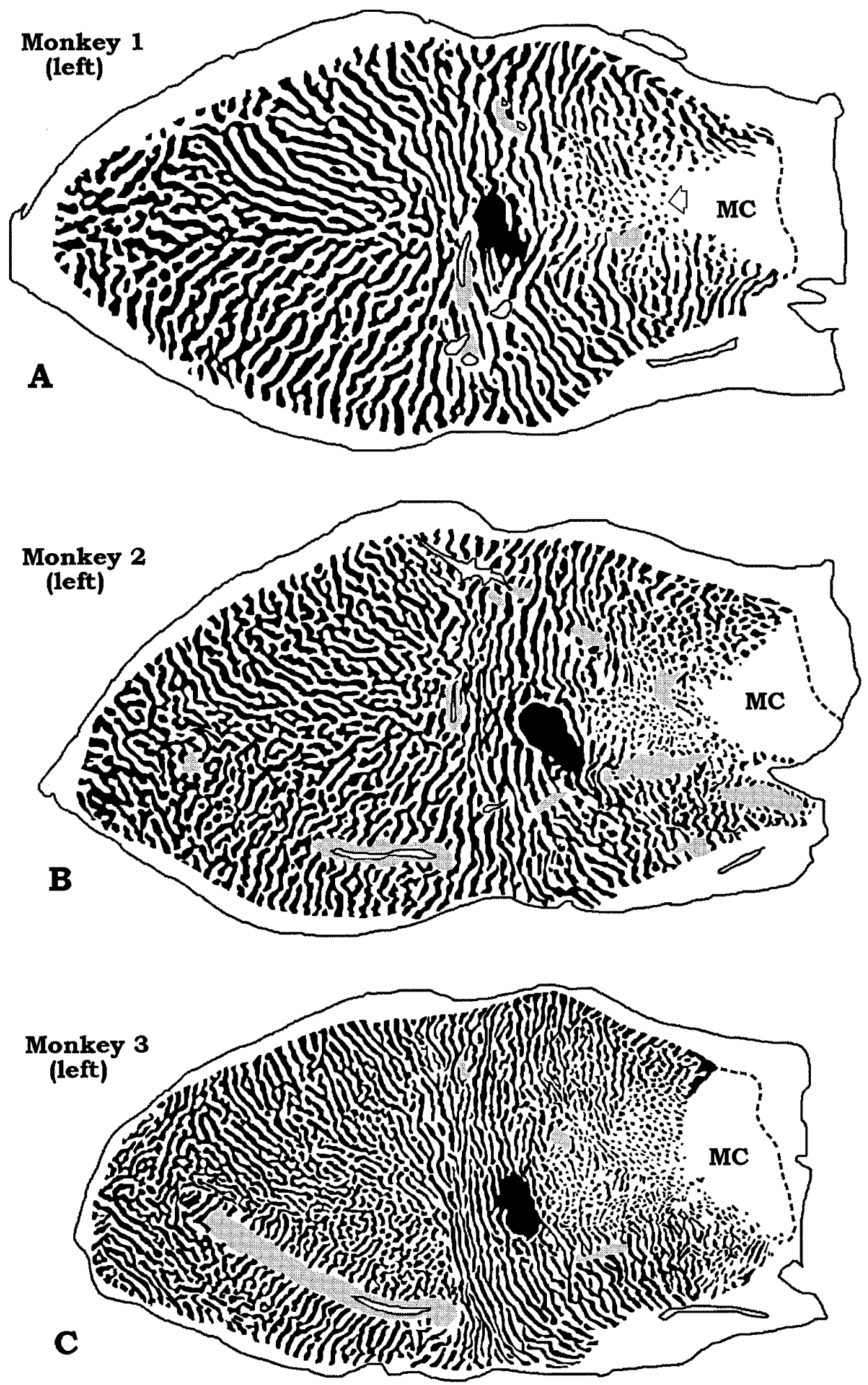

Figure 3. Drawings of the montages in Figure 2. The gray stippling indicates regions where the columns were unreconstructable. $M C$, Monocular crescent The ipsilateral eye's ocular dominance columns fragment into islands in the peripheral field representation along the horizontal meridian $(A$, open arrow). In this region, the contralateral eye predominates strongly. On the operculum, islands also appear but in a more scattered distribution. When they occur on the operculum, they are more likely to serve the ipsilateral eye than the contralateral eye, just as in the periphery (e.g., on the operculum there are more dark islands in $A-C$ and more pale islands in $D-F$ ). This suggests that the contralateral eye predominates in the central visual field representation too, albeit to a lesser degree than in the periphery. 

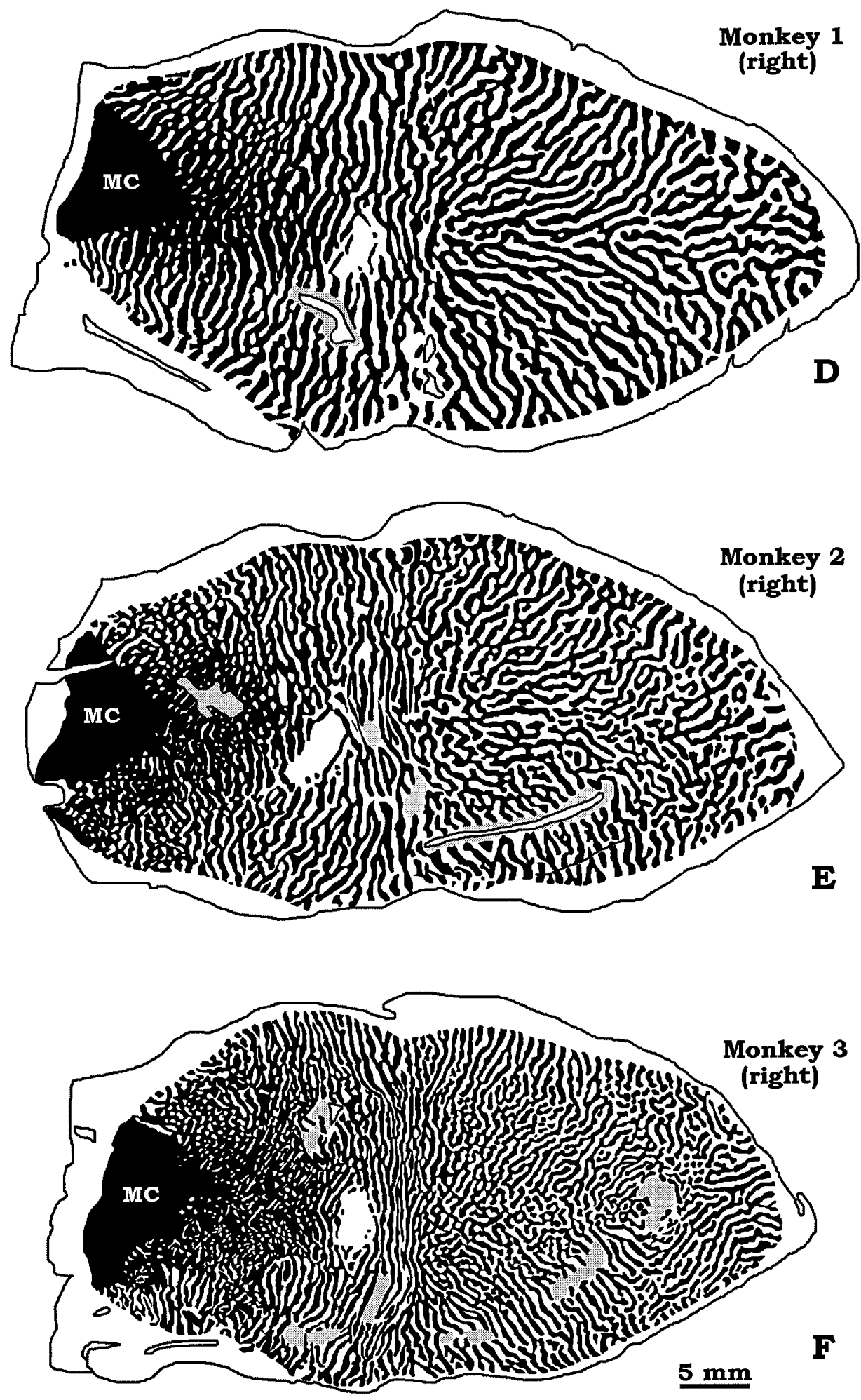

biased our drawings and, therefore, our area measurements in peripheral cortex. Third, the diaminobenzidine reaction product accumulates some distance from the $\mathrm{CO}$ catalyst, potentially expanding the dark columns beyond their true width. Fourth, a continuous sheet of dark $\mathrm{CO}$ activity remains at the base of layer $\mathrm{IV} c \beta$ after monocular enucleation (Horton, 1984). If the montage grazes the base of layer IVc $\beta$, it will favor the dark columns.
Because of these factors, we believe that negligible shrinkage of ocular dominance columns occurs after monocular enucleation in adults. This conclusion is consistent with a previous study of human striate cortex, showing nearly equal dark and light $\mathrm{CO}$ column areas as long as 10 and 23 years after monocular enucleation (Horton and Hedley-Whyte, 1984). Whatever shrinkage occurs is probably caused by transneuronal atrophy in layer IVc, 

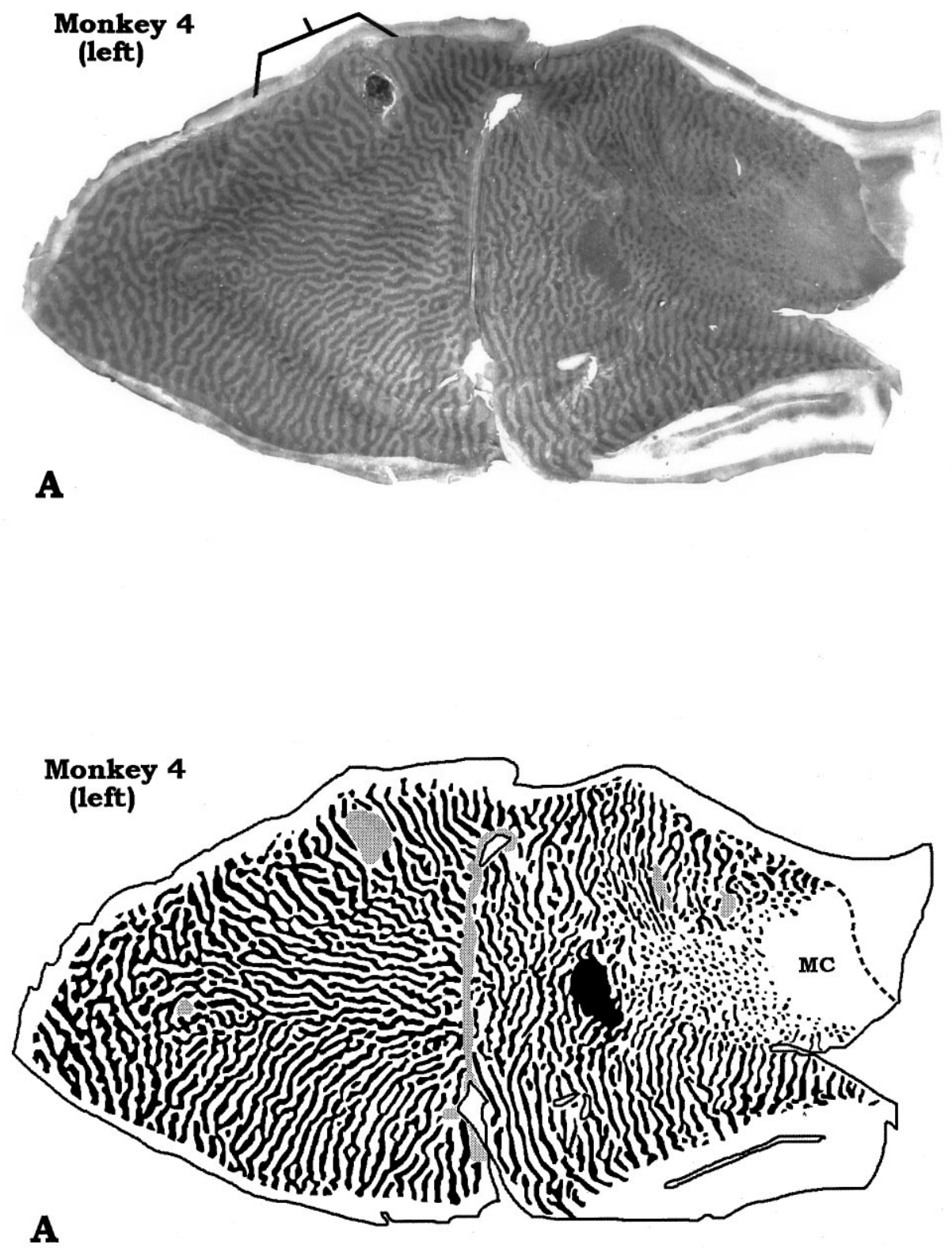

Figure 4. Montages from a macaque with striking enlargement of the ocular dominance columns in the foveal representation. The bracket shows another example of a minor anomaly: a region on the left operculum where the columns of the intact left eye (dark columns) appear relatively fragmented and shrunken. Note that in the corresponding region of the right operculum, the opposite is true.

(Figure continues next page.)

Figure 5. Drawings of the montages in Figure 4, showing the foveal "megacolumns." All macaques have larger columns in the foveal representation, but not all animals show this effect so conspicuously. In the right hemisphere, the dark columns serving the intact left eye appear relatively shrunken in the foveal representation. This provides another example of local imbalances in column areas that can occur on the operculum. This peculiarity tends to occur near the V1/V2 border or in the foveal area. $M C$, Monocular crescent.

(Figure continues next page.)

which gives rise to columns visible with Nissl stain (Haseltine et al., 1979), rather than sprouting of axon terminals serving the intact eye.

\section{Imbalance between columns serving the contralateral and ipsilateral eye}

LeVay et al. (1985) observed that from the blind spot to the monocular crescent $\left(18^{\circ}\right.$ to $\left.60^{\circ}\right)$, the ipsilateral eye's columns become increasingly attenuated and fragmented. This effect is most evident along the horizontal meridian, where they break into islands (see open arrow, Fig. $3 A$ ). As a result, columns driven by the contralateral eye occupy a greater fraction of peripheral cortex than columns of the ipsilateral eye. This imbalance was also seen by Florence and Kaas (1992) and Rosa et al. (1992). In our mosaics, from the "hinge" to the periphery $\left(\sim 8^{\circ}\right.$ to $\left.60^{\circ}\right)$, the contralateral eye/ipsilateral eye ratio of column areas was 63:37. This figure was derived from Table 2 by averaging the percentages 

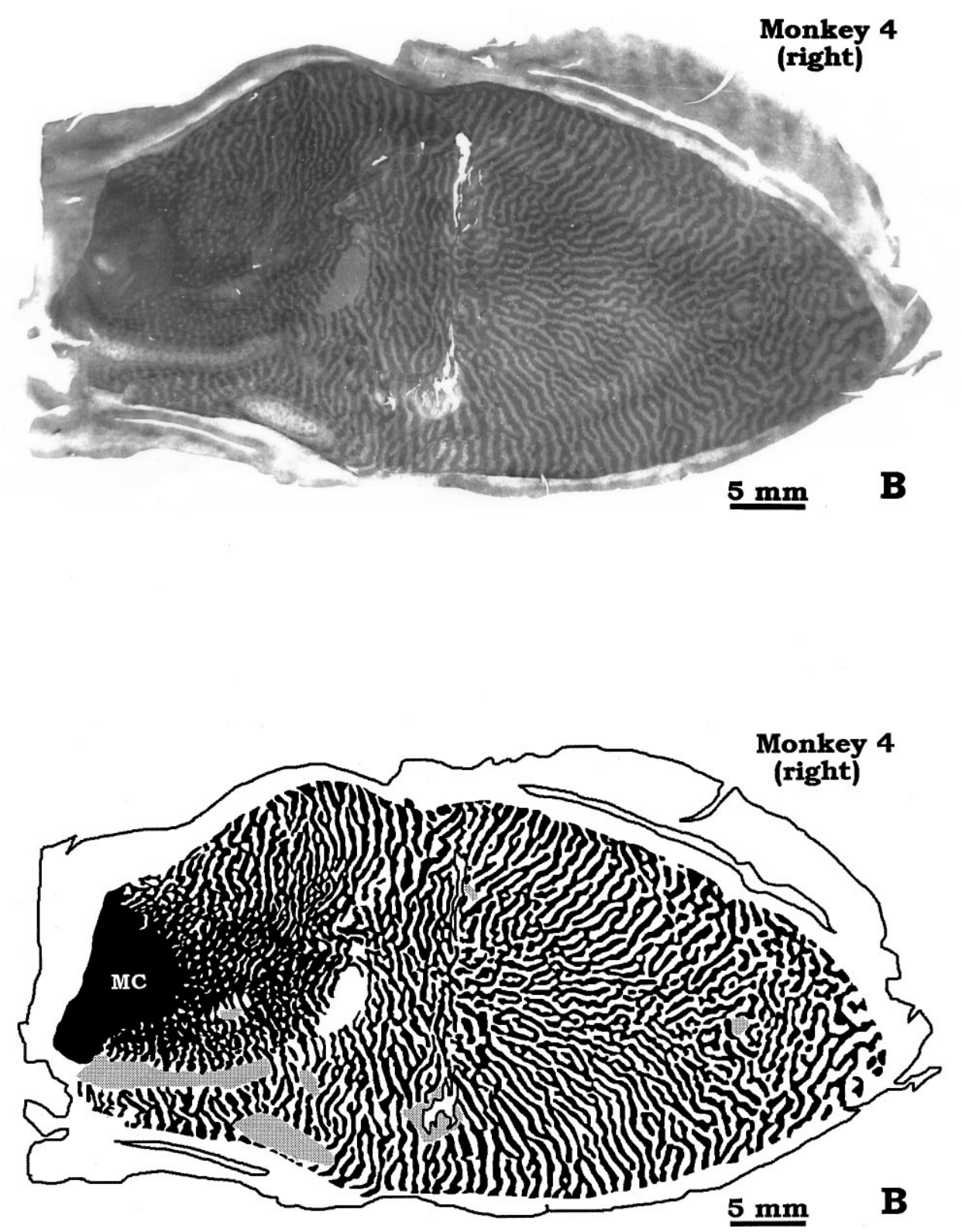

for column areas serving the contralateral eye and the ipsilateral eye in the periphery.

We were curious whether this imbalance in contralateral/ipsilateral eye columns extended onto the operculum. Averaging the percentages of opercular column areas, we found a ratio of 52:48, indicating mild predominance of the contralateral eye, even for the central field representation $\left(0^{\circ}\right.$ to $\left.8^{\circ}\right)$. The difference was modest and was not evident in every specimen. For several reasons, however, we were inclined to regard it as genuine. First, the confounding issue of bias in favor of the dark columns (reviewed above) cancels out, because the contralateral eye's columns are dark in half of the cases and light in half of the cases. Second, looking at the opercular portions of the mosaics, occasional islands that occur are more apt to belong to the ipsilateral eye, echoing the tendency in the periphery. Third, in a recent study of column mosaics in newborn macaques, labeled using $\left[{ }^{3} \mathrm{H}\right]$ proline rather than $\mathrm{CO}$, a slight predominance of the contralateral eye in opercular cortex was also found (Horton and Hocking, 1996).
In local areas of the column mosaics, one eye sometimes predominates strongly over the other, even on the operculum (see bracket, Figs. $2 A, 4 A$ ). We do not know why these local irregularities occur, but they could be mistaken easily for a deprivation effect if isolated from the context of the whole mosaic. This caveat is relevant to optical imaging studies, which typically sample only a small area of cortex.

\section{DISCUSSION}

In 1980, we began flat-mounting striate cortex by cutting off the operculum, splitting the calcarine fissure, and freezing the pieces against a glass slide to create large, smooth surfaces for sectioning. A practical motive, paramount to a graduate student, was to abbreviate the onerous chore of microtoming. Flat-mounting means fewer sections, with huge savings in time, slides, boxes, solutions, etc. For example, LeVay's original reconstruction required cutting more than 1000 serial horizontal sections through the intact occipital lobe. In the present study, less than 40 sections 
were cut from each flat-mount. Originally, another motive to prepare flat-mounts was to examine the two-dimensional array formed by $\mathrm{CO}$ patches in peripheral regions of striate cortex hidden from direct view, like the blind spot or monocular crescent (Horton, 1984). Dismembering the occipital lobe, however, had the drawback that the fragments did not fit back together and tissue was lost along the margins of the cuts. This problem was solved by Olavarria and Van Sluyters (1985) and Tootell and Silverman (1985), who showed that one could convert convoluted cortex to large tangential sheets by unfolding sulci and gyri. This approach has made it possible to flat-mount striate cortex in a single piece (Anderson et al., 1988; Rosa et al., 1988, 1992; Florence and Kaas, 1992). It reverses the sequence of steps taken by Daniel and Whitteridge (1961), who ingeniously derived a clay surface model of striate cortex by measuring cortical magnification factor, folded it up, cut serial parasagittal sections, and showed its close resemblance to the intact occipital lobe of the monkey.

Combining the flat-mounting technique with computer photomontaging has allowed us to reconstruct $96-99 \%$ of the column pattern in each animal with comparative ease. The principal finding to emerge is that a surprising degree of variation exists in the periodicity of ocular dominance columns in a sample of normal, adult macaques with similar V1 area. Along the V1/V2 border, the number of column pairs ranged between 101 and 154 and the average column width ranged from 670 to $395 \mu \mathrm{m}$, a factor of 1.7:1. These figures underestimate the true range, because they are based on examination of just six animals.

Flat-mounting striate cortex in both hemispheres of six animals permitted us to verify directly the accuracy of the original computer reconstruction done by LeVay et al. (1985) from serial sections of intact occipital lobe. Their rendition was faithful to the real, unfolded appearance of the ocular dominance columns and remains a breathtaking accomplishment. It was representative of an average macaque, with values for V1 area, perimeter, column widths, and number of column pairs that are close to the means of our reconstructions (Table 2). We can add only a few points to their description of the column pattern. First, the columns are slightly wider along the vertical meridian than the horizontal meridian (except for the contralateral eye's columns in the far periphery, which become enlarged by fragmentation of the ipsilateral eye's columns). Second, the fovea contains unusually large columns. This effect was not appreciated by LeVay et al. (1985), perhaps because the foveal region in their reconstruction was disrupted by relieving cuts, which gave it an excessively rounded appearance. Another possibility is that their animal had only modest enlargement of the foveal columns, because this phenomenon is quite variable. Third, the predominance they reported of the contralateral eye in peripheral cortex actually extends into the operculum, although it is a small effect $(\sim 52 \% / 48 \%)$, and dissipates the closer the columns get to the foveal representation. Fourth, their reconstruction was too elongated, with a major/minor axis of 2.4:1. This ratio was close to 2.0:1 in all of our specimens.

Löwel (1994) has reported that cats raised with strabismus have coarser ocular dominance columns, a finding questioned by Jones et al. (1996). Anderson et al. (1988) reconstructed the complete pattern of ocular dominance columns in four normal cats, but they did not examine intrinsic variation in column periodicity. Our findings suggest caution before ascribing changes in column width to various manipulations, because so much natural variation exists, at least among macaques. Of course, it is possible that our monkeys with the widest columns had microstrabismus. We examined our monkeys carefully for strabismus and found none.
However, one cannot exclude a small-angle deviation unless behavioral testing is done. Probably the most telling point is that ocular dominance columns form before birth in macaques, before strabismus can possibly have any influence on their pattern. Animals vary in ocular dominance column spacing and number right at birth (compare Figs. 2 and 3 in Horton and Hocking, 1996), indicating that this variability is inherent.

Tootell et al. (1988) compared the pattern of ocular dominance columns in opercular flat-mounts from two specimens of $M$. artoides and one specimen of $M$. fascicularis. They reported that "dominance strips are significantly wider in $M$. artoides than in $M$. fascicularis and this appears to be correlated with a larger brain size and a larger striate area." Given the variability we have found, it is premature to conclude that certain species of macaques have wider columns or that column width correlates with striate area in macaques. In striate cortex of $M$. mulatta, just as in $M$. fascicularis, there is a wide range in the number of sets of columns and in their spacing from animal to animal (Horton and Hocking, in press). We have no explanation for this variability, because we do not understand the factors controlling column dimensions. It would be interesting to examine the patterns of ocular dominance columns in identical twin macaques.

The mammalian cortex is subdivided into a multitude of functional areas, each containing its own columnar architecture. Little attention has been paid to the issue of variation from animal to animal in the periodicity and dimensions of columns in any given cortical area. Ocular dominance columns are among the easiest columns to label in the entire brain and, therefore, provide an excellent means for assessing natural variation in column size. Our data indicate that the width of ocular dominance columns can vary nearly twofold from one animal to the next. This finding has implications for other systems of columns in V1. Hubel and Wiesel (1977) have proposed that striate cortex is diced into modules, comprised of overlapping cycles (called hypercolumns) for each type of column. For these columns to remain in register, i.e., to express a complete set of values within each module, they must be sized proportionately. For example, one would predict that a macaque with especially wide ocular dominance columns would also have wider orientation columns. Unfortunately, we have no data pertaining to orientation columns in our animals, but we have counted the density of $\mathrm{CO}$ patches, which fit in register with the ocular dominance columns (Horton and Hubel, 1981). As expected, animals with thin ocular dominance columns have smaller, more numerous patches, whereas the opposite is true in animals with wide columns. For example, the density of $\mathrm{CO}$ patches on the operculum of Monkey 3 was well over twice the density of patches on the operculum of Monkey 1 (not illustrated). This finding is consistent with the wide range in total blobs per hemisphere found by Purves and LaMantia (1993) in their survey of normal rhesus monkeys.

Variation in ocular dominance column periodicity should also be reflected in the clumping patterns of geniculocortical afferents, the size of dendritic arbors of cortical cells, the periodicity of the lattice-like arrays of intrinsic horizontal connections (Rockland and Lund, 1983; Lund et al., 1993), and the patchiness of connections from V1 to other cortical areas. It would be of particular interest to learn whether animals with more pairs of ocular dominance columns tend to have more cycles of $\mathrm{CO}$ slabs (thick, pale, thin, pale) in V2. This might indicate whether relative column periodicity varies independently by cortical area, or whether it is a correlated, transcendent property of the entire cortex for any given brain. 


\section{REFERENCES}

Anderson PA, Olavarria J, Van Sluyters RC (1988) The overall pattern of ocular dominance bands in cat visual cortex. J Neurosci 8:2183-2200.

Daniel PM, Whitteridge D (1961) The representation of the visual field on the cerebral cortex in monkeys. J Physiol (Lond) 159:203-221.

Florence SL, Kaas JH (1992) Ocular dominance columns in area 17 of old world macaque and talapoin monkeys: complete reconstructions and quantitative analyses. Vis Neurosci 8:449-462.

Haseltine EC, DeBruyn EJ, Casagrande VA (1979) Demonstration of ocular dominance columns in Nissl-stained sections of monkey visual cortex following enucleation. Brain Res 176:153-158.

Hess DT, Edwards MA (1987) Anatomical demonstration of ocular segregation in the retinogeniculocortical pathway of the new world capuchin monkey (Cebus apella). J Comp Neurol 264:409-420.

Horton JC (1984) Cytochrome oxidase patches: a new cytoarchitectonic feature of monkey visual cortex. Philos Trans R Soc Lond [Biol] 304:199-253.

Horton JC, Hedley-Whyte ET (1984) Mapping of cytochrome oxidase patches and ocular dominance columns in human visual cortex. Philos Trans R Soc Lond [Biol] 304:255-272.

Horton JC, Hocking DR (1996) An adult-like pattern of ocular dominance columns in striate cortex of newborn monkeys prior to visual experience. J Neurosci 16:1791-1807.

Horton JC, Hocking DR (1997) Myelin patterns in V1 and V2 of normal and monocularly enucleated monkeys. Cereb Cortex, in press.

Horton JC, Hubel DH (1981) Regular patchy distribution of cytochrome oxidase staining in primary visual cortex of macaque monkey. Nature 292:762-764.

Horton JC, Dagi LR, McCrane EP, de Monasterio FM (1990) Arrangement of ocular dominance columns in human visual cortex. Arch Ophthalmol 108:1025-1031.

Houghton P (1988) Virus-free African cynomolgus macaques. Lab Animal 17:36-40.

Hubel DH, Wiesel TN (1969) Anatomical demonstration of columns in the monkey striate cortex. Nature 221:747-750.

Hubel DH, Wiesel TN (1977) Functional architecture of macaque monkey visual cortex. Proc R Soc Lond [Biol] 198:1-59.

Hubel DH, Wiesel TN, LeVay S (1977) Plasticity of ocular dominance columns in monkey striate cortex. Philos Trans R Soc Lond [Biol] 278:377-409.

Jones DG, Murphy KM, Van Sluyters RC (1996) Spacing of ocular dominance columns is not changed by monocular deprivation or strabismus. Invest Ophthalmol Vis Sci 37:1964.

LeVay S, Wiesel TN, Hubel DH (1980) The development of ocular dominance columns in normal and visually deprived monkeys. J Comp Neurol 191:1-51.
LeVay S, Connolly M, Houde J, Van Essen DC (1985) The complete pattern of ocular dominance stripes in the striate cortex and visual field of the macaque monkey. J Neurosci 5:486-501.

Löwel S (1994) Ocular dominance column development: strabismus changes the spacing of adjacent columns in cat visual cortex. J Neurosci 14:7451-7468.

Lund JS, Yoshioka T, Levitt JB (1993) comparison of intrinsic connectivity in different areas of macaque monkey cerebral cortex. Cereb Cortex 3:148-162.

Obermayer K, Blasdel GG (1993) Geometry of orientation and dominance columns in monkey striate cortex. J Neurosci 13:4114-4129.

Olavarria J, Van Sluyters RC (1985) Unfolding and flattening the cortex of gyrencephalic brains. J Neurosci Methods 15:191-202.

Purves D, LaMantia A (1993) Development of blobs in the visual cortex of macaques. J Comp Neurol 334:169-175.

Rockland KS, Lund JS (1983) Intrinsic laminar lattice connections in primate visual cortex. J Comp Neurol 216:303-318.

Roe AW, Ghose GM, Smith EL, Chino YM, Ts'o DY (1995) Alterations in striate cortical ocular dominance columns in anisometropic amblyopia. Soc Neurosci Abstr 21:689.9.

Rosa MGP, Gatass R, Fiorani M (1988) Complete pattern of ocular dominance stripes in V1 of a new world monkey, Cebus apella. Exp Brain Res 72:645-648.

Rosa MGP, Gattass R, Fiorani M, Soares JGM (1992) Laminar, columnar and topographic aspects of ocular dominance in the primary visual cortex of Cebus monkeys. Exp Brain Res 88:249-264.

Tieman SB, Tumosa N (1996) Alternating monocular exposure increases the spacing of ocularity domains in area 17 of cats. Invest Ophthalmol Vis Sci 37:1965.

Tootell RBH, Silverman MS (1985) Two methods for flat-mounting cortical tissue. J Neurosci Methods 15:177-190.

Tootell RBH, Hamilton SL, Silverman MS, Switkes E (1988) Functional anatomy of macaque striate cortex. I. Ocular dominance, binocular interactions, and baseline conditions. J Neurosci 8:1500-1530.

Trusk TC, Kaboord WS, Wong-Riley M (1990) Effects of monocular enucleation, tetrodotoxin, and lid suture on cytochrome oxidase reactivity in supragranular puffs of adult macaque striate cortex. Vis Neurosci 4:185-204.

Van Essen DC, Newsome WT, Maunsell JHR (1984) The visual field representation in striate cortex of the macaque monkey: asymmetries, anisotropies, and individual variability. Vision Res 24:429-448.

Wiesel TN, Hubel DH, Lam DMK (1974) Autoradiographic demonstration of ocular-dominance columns in the monkey striate cortex by means of transneuronal transport. Brain Res 79:273-279.

Wong-Riley M (1979) Changes in the visual system of monocularly sutured or enucleated kittens demonstrable with cytochrome oxidase histochemistry. Brain Res 171:11-28. 